\title{
La cooperación regional institucionalizada y los retos de la crisis estructural en América Latina
}

\author{
josé Raúl Perales
}

José Raúl Perales. licenciado en CienCIAS POLÍTICAS dE LA UNIVERSIDAD dE PUERto Rico. Candidato a Doctor EN CIENCias Políticas de la Universidad de Michigan (AnN Arbor). 
El resurgimiento de los esfuerzos integracionistas en América Latina desde principios de la década de los noventa, ha dado lugar a una discusión extensa sobre los retos, oportunidades y beneficios del libre comercio en la región. Sin embargo, la manera y la oportunidad que estos esfuerzos, en tanto instancias de cooperación regional institucionalizada han surgido, no ha sido suficientemente abordada por teóricos latinoamericanos de relaciones internacionales. Este ensayo presenta un marco analítico y conceptual, basado en la lógica de acción colectiva y de ganancias absolutas en las 
relaciones interestatales, así como en teoría de regímenes, para reflexionar sobre la integración latinoamericana desde una perspectiva sistémica de las relaciones internacionales. Se presenta un examen sobre la convergencia entre las preferencias políticas de los estados latinoamericanos a fines de la década de los ochenta, tanto en términos substantivos como en cuanto a las reglas del juego, que permite entender cómo la integración se convirtió, para muchos gobiernos de la región, en una herramienta para lograr las metas individuales de cada estado. 


\section{La cooperación regional institucionalizada y los retos de la crisis estructural en América Latina*}

Pocas instancias en el avance hacia niveles superiores de desarrollo económico entre las regiones sujetas a este proceso se pueden comparar con la encrucijada material, política y social en la que América Latina y el Caribe se encuentran actualmente. Desde hace una década, la región ha venido confrontando una crisis de discontinuidad de los modelos políticos y económicos tradicionales que informaron estos procesos durante las décadas posteriores a la Segunda Guerra Mundial. ${ }^{1}$ Ante esta coyuntura del proceso desarrollista latinoamericano, las «fórmulas mágicas» autóctonas para el avance de los intereses nacionales se han vuelto relativamente obsoletas, pero aún ningún sustituto para estos modelos, si es que tal sustituto es deseable, ha podido constituirse. Esta situación, que algunos analistas consideran perjudicial y crítica (Stallings, 1994; Roett, 1991) mientras otros la perciben como sumamente oportuna (Rojas Aravena, 1993; Haggard, 1990), conlleva un delicado balance entre la capitulación final de la racionalidad de sustitución de importaciones, a favor de una apertura sustancial de los mercados nacionales a la competencia y el comercio internacional, ${ }^{2}$ así como una reestructuración institucional significativa en los gobiernos nacionales para dar paso a sistemas políticos democráticos. La presencia de profundas crisis fiscales y de inestabilidad macroeconómica, la ausencia de inversiones de capital extranjero (y aún doméstico), la fuga de capitales, así como las reducciones en el gasto público, unidas al deterioro de los indicadores sociales y a las crisis de gobernabilidad a través del continente, proveyeron las condiciones coyunturales a fines de los ochenta para repensar los modelos latinoamericanos de desarrollo. Durante este período, las respuestas autóctonas a la crisis fueron temporalmente descartadas como resultado de las exigencias inmediatas de estabilizar las economías de la región. La fórmula neoliberal

\footnotetext{
* Una versión preliminar de este trabajo fue presentada en la XX Reunión de la Asociación de Estudios del Caribe (CSA) en Willemstad, Curacao, 23 al 25 de mayo de 1995 . Agradezco los comentarios y sugerencias de Ted Hopf, Denise DeGarmo, Rita Giacalone, Francisco Catalá y Juan Lara. Cualquier omisión o fallas en este trabajo son responsabilidad Exclusiva del autor. ${ }^{2}$ Para un balance y examen crítico de los proyectos de reforma económica latinoamericana, véase Inter American Development Bank, 2997 Social and Economic Progress Report - Latin America: A Decade of Reforms (Washington, D.C.: Inter American Development Bank / Johns Hopkins University Press, 1997.) Para un recuento analítico de las transformaciones socioeconómicas en América Latina como resultado de las reformas neoliberales, véase Sebastian Edwards, Crisis and Reform in Latin America (New York: The World Bank / Oxford University Press, 1995.)

${ }^{2}$ Estos asuntos han cobrado cierta importancia como parte de un mayor énfasis en el campo de política comparada de la importancia de las ideas en las cúpulas gubernamentales para entender los determinantes, y a veces los resultados, de los programas económicos. Véase Kathryn Sikkink, ldeas and Institutions: Developmentalism in Brazil and Argentina (Ithaca NY: Cornell University Press, 1990); Peter Hall (ed.), The Political Power of Economic Ideas (Princeton, NI: Princeton University Press, 1992); Judith Goldstein, Ideas and American Trade Policy (Princeton, N): Princeton University Press, 1994); Sylvia Maxfield y Miguel Angel Centeno,'The Marriage of Finance and Order: Changes in the Mexican Political Elite», Journal of Latin American Studies 24: 57-85; y Jorge Dominguez (ed.), Technopols: Freeing Politics and Markets in Latin America in the 1990 s (University Park, PA: Pennsylvania State University Press, 1997.)
} 
proveniente de agencias internacionales como el Banco Mundial y el Fondo Monetario Internacional suministraron una respuesta inmediata a la crisis de inestabilidad.

Es precisamente dentro de este contexto histórico, cuando Latinoamérica ha visto el resurgimiento de esquemas de integración. Nuevos organismos así como regímenes existentes han sido activados a través de toda la región, en un intento por alcanzar una «inserción exitosa en la economía mundial» (CEPAL, 1994a). La movilización política y económica hacia la regionalización económica ha sido considerada en general como la confluencia de dos desarrollos históricos en particular: por un lado, la puesta en práctica de programas comunes de macroestabilización económica a través de América Latina, lo cual convirtió la coordinación macroeconómica en una condición, más que deseada, realmente necesaria para lograr cualquier grado de crecimiento económico (CEPAL 1992); y por otro lado, la formación de foros institucionalizados para la cooperación y la negociación internacional como resultado de Contadora y el Grupo de Río (Frohmann, 1990). La búsqueda de respuestas regionales a dos problemas de gran urgencia -el escalamiento y la proliferación del conflicto bélico en Centroamérica y la crisis de la deuda externa- proveyó una singular experiencia de cooperación regional, que ulteriormente muy bien pudo haber renovado la expectativa de que una de las cuestiones estructurales más importantes de la historia económica de América Latina desarrollo- también pudiera ser resuelta a través de esfuerzos regionales.

La lógica de la liberalización económica, como parte integral de los programas de macroestabilización económica puestos en marcha a partir de las recomendaciones del Fondo Monetario Internacional, a su vez impartió un estimulo estructural para un nuevo modelo de integración regional. Este estímulo, irónicamente, resultó ser un elemento decisivo para el regionalismo económico en la medida en que los programas neoliberales de ajuste estructural requieren de mercados abiertos, fomento a las exportaciones y reducciones en barreras comerciales. Las nuevas reglas del juego facilitaron posteriormente la formación de los elementos económicos, financieros y empresariales necesarios para la búsqueda de economías de escala en algunos países. Finalmente, la Iniciativa para las Américas inaugurada por el Presidente de Estados Unidos George Bush en 1990 abrió nuevas posibilidades para la cooperación hemisférica. En cierto modo, ésta aseguró la confianza de gran parte de los líderes latinoamericanos sobre la nueva actitud estadounidense hacia la concertación regional, sobre todo en el área comercial.

Sin embargo, la manera en que las instituciones regionales latinoamericanas que surgieron como respuesta a los retos económicos internacionales aún debe ser explicada. La integración, según nota Peter Smith, conlleva la creación de una entidad nueva capaz de proveer un marco de referencia sólido para la coordinación de políticas macroeconómicas y para el intercambio de capitales, bienes y servicios. La integración también conlleva un alto grado de 
expectativas, metas e intereses comunes (Smith, 1993). Esto representa un salto cualitativo dentro del tema de la cooperación internacional, la cual une a dos o más actores con un asunto o preocupación en común, a través de negociaciones, en ocasiones a lo largo de un período de tiempo determinado y con una agenda específica. La idea del efecto spillover que comúnmente se aborda en estudios sobre el desarrollo de la Comunidad Europea (Pierson, 1995) no puede proveer un vínculo causal que de cuenta de los motivos por los cuales los Estados latinoamericanos se interesaron en crear, o reactivar, regímenes de integración luego de Contadora. De hecho, aún a pesar del ejemplo de la Comunidad Europea, el influyente papel de la Comunidad en el surgimiento de la modalidad particular de regionalización económica latinoamericana tendría un significado muy limitado si no hubiera una motivación política, y una evolución subsiguiente, de la cooperación institucionalizada. Aún queda por verse, tomando en consideración los fracasos anteriores en la agenda integracionista (y dejando a un lado las aspiraciones históricas de un continente unido), por qué los Estados latinoamericanos han optado por aplicar la misma forma institucional a exigencias económicas estructurales de una naturaleza diferente.

Este ensayo intenta proveer una perspectiva teórica sobre esta pregunta mediante un abordaje de dos condiciones estructurales que conforman el lugar que ocupa Latinoamérica en el sistema internacional contemporáneo la transición y consolidación de regímenes democráticos y la búsqueda de nuevas respuestas a los desafíos del desarrollo. En particular, se ofrecen ideas que permiten conciliar lo que aparentan ser dos lógicas contradictorias en el espectro político latinoamericano. La presunta contradicción radica en el hecho de que la primera «condición» (democratización) requiere de una dispersión del poder político fuera del ámbito exclusivo del ejecutivo, mientras que la segunda «condición» (reestructuración económica) aparentemente exige lo opuesto. ${ }^{3}$ El énfasis de esta discusión gira en torno a la variable sistémica de la integración regional, la cual asume una perspectiva analítica intergubernamental de los esfuerzos integracionistas. ${ }^{4}$ Esta premisa se ubica al centro de un debate teórico dentro del estudio de las relaciones internacionales, en el cual críticos de la escuela neorrealista (Baldwin, 1993; Putnam, 1988) han desafiado la conceptualización tradicional de los Estados como actores de carácter unitario y racional. En términos de los estudios sobre la integración, esta caracterización conlleva serias implicaciones. La definición de los Estados nacionales como actores racionales con interés propio implica que los regímenes de integración han de conver-

\footnotetext{
${ }^{3}$ Este planteamiento queda evidenciado si se observa la cantidad de medidas de reforma económica que han sido implantadas como resultado de decretos presidenciales, en países como Argentina, Bolivia, Brasil, y Venezuela. A su vez, la integración regional latinoamericana misma es el resultado de iniciativas políticas a nivel del ejecutivo, con limitado desarrollo institucional y escasa participación de otras ramas gubernamentales. Sobre este último punto, véase Manzetti (1993) y Pastor (1994).
} 
tirse en foros de negociación interestatal, donde la forma de canalizar efectivamente las distintas presiones domésticas de carácter social y político es a través de los representantes y negociantes de los Estados (Pierson, 1995). Este ensayo no asume tal postura, ya que, usando la terminología de Robert Putnam, es precisamente el carácter de «doble nivel» (específicamente, en términos de presiones simultáneas a nivel doméstico e internacional) de los procesos de integración lo que hace de éstos una empresa difícil de culminar. ${ }^{5}$ Sin embargo, en aras de preservar cierta parsimonia analítica, el uso aquí del acercamiento «estatista» permite una mejor representación del rol de las estructuras en el en el surgimiento de la cooperación institucionalizada entre los Estados latinoamericanos.

Por consecuencia, este ensayo aplica elementos de la lógica de teoría del juego contenida en el dilema sobre ganancias relativas versus ganancias absolutas y sobre institucionalismo para comprender la cooperación internacional y la formación de regímenes en el caso de la evolución de los esquemas de integración a través de América Latina. Como se verá más adelante, el surgimiento de dichos regímenes ha sido el resultado de una convergencia parti-

4 El estudio político de los procesos de integración regional ha sido marcado por una controversia entre dos escuelas analíticas, ambas interpretaciones del fenómeno europeo. Una es la llamada intergubernamentalista, que plantea que el avance de la integración regional es el resultado de negociaciones entre los países que componen el esquema integracionista, donde cada miembro negocia la instancia regional a partir de intereses nacionales concretos. Bajo este marco interpretativo de la integración, los países miembros de un esquema establecen la agenda del proceso de regionalización, y por lo mismo son capaces de detener y obstaculizar el avance de la integración si ésta no corresponde a sus intereses. Entre los trabajos «intergubernamentalistas" más sobresalientes figuran Andrew Moravcsik, "Preferences and Power in the European Community: A Liberal Intergovernmentalist Approach» Uournal of Common Market Studies 31 [1993]: 473-524); Moravcsík, "Negotiating the Single European Act: National Interests and Conventional Statecraft in the European Community" (International Organization 45 [1991]: 19-56); y Joseph Grieco «Systemic Sources of Variation in Regional Institutionalization in Western Europe, East Asia, and the Americas" en Edward Mansfield y Helen Milner (eds.), The Polltical Economy of Regionalism (New York: Columbia University Press, 1997). Por el contrario, el funcionalismo plantea que el avance de la integración regional es el resultado de un proceso donde una institución regional, fundada por un grupo de países con un propósito específico, adquiere relevancia como instancia de cooperación a medida que los resultados positivos de la primera agenda motivan a los actores nacionales a expandir la cooperaciôn hacia otras áreas previamente excluidas del acuerdo integracionista. De esta manera, la institución regional adquiere vida propia, y hasta llega a tener suficiente coherencia y autonomía como para sentar una agenda regional a la cual los estados nacionales deben responder. Entre los trabajos más relevantes de la escuela funcionalista figuran Ernst B. Haas, Beyond the Nation-State: Functionalism and International Organization (Stanford, CA: Stanford University Press, 1964); y más recientemente, Dorette Corbey, "Dlalectical Functionalism: Stagnation as a Booster of European Integration" (International Organization 49 [1995]: 253-284.)

5 Robert Putnam, "Diplomacy and Domestic Politics: The Logic of Two-Level Games» (International Organization 43 [1988]: 427-460.) El modelo de los juegos de "doble nivel» enfatiza el tipo de negociación interinacional que un líder debe lograr para así poder satisfacer dos audiencias a las que debe responder simultáneamente: una internacional (con la cual está negociando directamente), y una doméstica (la cual le impone límites al carácter y al alcance de los acuerdos que el líder negocia en el extranjero.) Las dos audiencias tienen intereses potencialmente coincidentes en algunas áreas; el interés del líder ante una negociación internacional por tanto es aumentar la cantidad de intereses superpuestos entre sus dos audiencias (lo que en inglés se conoce como "win-set») mientras que a la vez pueda reducir el "wir-set» de su contraparte en la negociación. Los líderes frecuentemente se valen de enlaces políticos en otras palabras, vincular temas de otras áreas a la negociación internacional en cuestiön- para expandir el conjunto de su «win-set». Sobre este punto, véase Susanne Lohmann, "Linkage Politics" Oournal of Conflict Resolution 41 [1997]: 38-67.) Para una aplicación del modelo a distintos tipos de negociación internacional, véase Peter B. Evans, Harold K. Jacobson, y Robert D. Putnam, Double-Edged Diplomacy: International Bargaining and Domestic Politics (Berkeley, CA: University of Califomia Press, 1993). 
cular de intereses y preferencias entre los participantes del esquema de integración, de manera que la coyuntura de constreñimientos económicos (estrategias de desarrollo bajo condiciones de deuda externa y reestructuración neoliberal) y de posibilidades políticas (el resurgimiento de gobiernos democráticos y los proyectos de reforma estatal) crean una situación en la cual los Estados han alterado el énfasis de sus relaciones internacionales de una perspectiva de ganancias relativas a una perspectiva de ganancias absolutas. Por lo tanto, la cooperación por vía de la integración no sólo sirve como un asunto político, inmediato para los gobiernos latinoamericanos (sobre todo en términos de manejo de crisis, de legitimidad y de credibilidad), sino también serviría como un paso significativo en la transformación del carácter de la política internacional en la región. Se espera que el siguiente ejercicio analítico permita una mejor conceptualización de un argumento de ganancias absolutas por vía de la integración que pudiera posteriormente servir como base para comparar determinantes de regímenes de integración entre países en desarrollo en otras regiones.

\section{Los desafíos del desarrollo en los noventa}

En su análisis sobre los requisitos del desarrollo económico en América Latina para los 1990, Bárbara Stallings (1994) observa que, contrario a décadas previas donde la cuestión desarrollista se consideraba predominantemente como un asunto de estructuras y capacidades domésticas, el contexto contemporáneo de la evolución del desarrollo contiene una paradoja fundamental. De hecho, "han ocurrido impactantes cambios a nivel internacional en el campo de la política, la economía y la seguridad, poniendo de cabeza al mundo que conocíamos desde hace medio siglo. El sentido común nos dice que estos cambios no pueden dejar de tener un impacto sobre las posibilidades de desarrollo, pero puesto que permitimos que nuestras herramientas conceptuales se enmohecieran no estamos, en consecuencia, en condiciones de analizar los mecanismos en sí mismos ni sus efectos. Tampoco podemos explicar cabalmente la respuesta ante estos cambios." 6

Estamos presenciando pues un cambio de paradigmas; un cambio en las herramientas conceptuales y en la realidad empírica que han definido la problemática desarrollista en América Latina durante las pasadas décadas. Variables domésticas e internacionales que tradicionalmente han jugado un papel secundario en las políticas desarrollistas surgen simultáneamente como obstáculos y como oportunidades para la conceptualización de un nuevo modelo de crecimiento y de transformación económica (tales como la competencia en mercados inter-

\footnotetext{
${ }^{6}$ Traducción del autor.
} 
nacionales, la estabilidad monetaria y la eficiencia en servicios). Sin embargo, la creación de dicho modelo ha quedado relativamente rezagada, en gran parte, por el fracaso de las fórmulas autóctonas (sobre todo la sustitución de importaciones y los primeros esfuerzos heterodoxos) y su incapacidad de generar niveles consistentes de desarrollo y una solución a las crisis en la balanza de pagos de los años 1980. De hecho, las fórmulas neoliberales de estabilización macroeconómica provenientes de instituciones financieras internacionales proveyeron a fines de los 80 principios de los 90 una alternativa inmediata, si bien dolorosa, a la crisis macroeconómica. Aún cuando en este ensayo no se presenta un análisis más elaborado sobre los efectos específicos de estas políticas, es sin embargo necesario conveniente señalar dos consecuencias relevantes del proceso de reajuste neoliberal: la «internacionalización» del desarrollo latinoamericano y la transformación de la agenda desarrollista en un bien colectivo negociable en el espacio internacional. ${ }^{7}$

Ha sido comúnmente reconocido que entre los factores decisivos en el agotamiento de cualquier estrategia de desarrollo figuran las presiones del sistema internacional (Gereffi, 1994). El síndrome de la «década perdida» en América Latina evidencia este reclamo, toda vez que durante ese período las políticas y fórmulas de crecimiento económico concebidas en la región misma colapsaron como resultado de la crisis de la deuda externa. El neoliberalismo adjudica responsabilidad sobre este fracaso de los Estados latinoamericanos en proveer un modelo adecuado de desarrollo económico a deficiencias institucionales. Según esta perspectiva, la estructura misma de los Estados latinoamericanos, específicamente las burocracias domésticas incrementadas, es incapaz de fomentar niveles de crecimiento económico sostenido y mucho menos en momentos de crisis. ${ }^{8}$ En términos de política económica, las decisiones nacionales se vieron aún más constreñidas por las estrategias ortodoxas de reforma impuestas por las agencias financieras internacionales. Aún cuando el desarrollo económico había sido siempre materia de diseño e implementación estatal, la urgencia de las políticas de ajuste inhibieron la viabilidad de estrategias nacionales de desarrollo patrocinadas por los Estados.

7 El término «bien colectivon se refiere a aquéllos de cuya provisión se beneficia la totalidad de los componentes de un sistema social. Como tal, los bienes colectivos son el resultado del esfuerzo de un grupo concentrado de personas o entidades, cuyo resultado redunda en un beneficio generalizado que no hace excepciones, aún en el caso de actores sociales que no hayan cooperado en la provisión del bien. El análisis clásico de este tipo de dinámica social se encuentra en Mancur Olson, The Logic of Collective Action (Cambridge, MA: Harvard University Press, 1965). Para una aplicación del concepto a la esfera internacional, véase John Conybeare, "Public Goods, Prisoner's Dilemmas, and International Political Economy," International Studies Quarterly 28(1): 5-22. [1984].

${ }^{8}$ La literatura sobre la ineptitud del estado latinoamericano ante los retos de la crisis de la deuda y la reestructuración económica es extensa. Un buen resumen analítico de los retos de la reforma institucional se encuentra en Sebastian Edwards, Crisis and Reform in Latin America (New York: Oxford University Press / World Bank, 1995). Sin embargo, para una crítica y análisis institucional del Estado en América Latina, véase Albert Fishlow, "The Latin American State», Journal of Economic Perspectives 4 (verano 1990): 61-74; y «La tentación del Estado: demandas y experiencias»- Nueva Sociedad (tema central) 104 (1989): 88-171. 
La interrogante del desarrollo por tanto se convirtió en una cuyas respuestas habrían de ser halladas no sólo en el ámbito político o económico nacional, sino en las condiciones y posibles oportunidades que ofrece la comunidad financiera y económica internacional. El énfasis en la liberalización comercial, la privatización, la Inversión extranjera y la reducción a las trabas comerciales como estaban enfatizadas en la «receta» neoliberal desplazó una parte significativa del foco de atención de la negociación política del espacio doméstico al internacional. ${ }^{9}$ Aún cuando esta situación pudiera haber reducido la autonomía nacional en cuestión de decisiones económicas, a su vez este cambio ha proporcionado una nueva oportunidad y un nuevo contexto para el surgimiento de una mayor y más fuerte cooperación entre los Estados latinoamericanos. En este sentido, la tendencia global hacia la liberalización del comercio podría ser considerada como un impulso inicial favorable para la cooperación en otros temas económicos, en la medida en que estimula la coordinación de políticas macroeconómicas en asuntos de comercio y flujo de capitales entre las economías en proceso de reajuste (CEPAL, 1992).

Se desprende de este argumento que, una vez que la búsqueda y la provisión de un bien colectivo requiere de mediación institucional fuerte para evitar el problema del free-rider ${ }^{10}$ (Olson, 1982), la internacionalización del desarrollo como bien colectivo implica que el Estado nacional por su propia cuenta es incapaz de movilizar (¿coaccionar?) a todos los actores necesarios para la provisión óptima de dicho bien. En otras palabras, las opciones y estrategias desarrollistas actuales tienen que contar con un elemento de internacionalización de la agenda económica en un contexto donde la cooperación internacional, luego del final de la Guerra Fría, no se basa en valor estratégico sino en reglas de juego basadas en el libre mercado, la competencia y la interdependencia económica global. En este contexto, dados los incentivos para desertar de compromisos internacionales aludidos anteriormente, la institucionalización de la cooperación internacional es de suma importancia para los países en desarrollo, especialmente si tal cooperación busca mejor acceso a mercados internacionales.

La experiencia histórica de los proyectos desarrollistas en América Latina evidencia la relación entre estrategias de desarrollo y arreglos estatistas de las coaliciones dentro de la

\footnotetext{
9 Para una discusión sobre esta transformaciôn, véase CEPAL (1992), CEPAL (1994a.), y CEPAL (1994b.)

${ }^{10}$ Véase Olson (1965), nota al calce \#7. El problema del «free ridern se trata precisamente de que, ante la naturaleza abarcadora de los bienes colectivos, no existen incentivos individuales para que un actor social coopere en la provisión del bien colectivo, sobre todo porque aûn si no coopera recibirá los beneficios de dicho bien colectivo. La lógica de esta dinámica social ha permitido a diversos analistas comprender mejor los obstáculos en materia de cooperación interestatal, ya que la naturaleza anárquica del sistema internacional (donde la autoridad suprema radica en los Estados que lo compo. nen) facilita el incumplimiento de compromisos de cooperación, sobre todo porque los Estados desertores resultan beneficiados (directa o indirectamente) de los logros de dicha cooperación. Véase Robert Axelrod, The Evolution of Cooperation (New York: Basic Books, 1984); Robert o. Keohane, After Hegemony: Cooperation and Discord in the World Political Economy (Princeton: Princeton University Press, 1984); y Kenneth Oye (ed.), Cooperation Under Anarchy (Princeton, N): Princeton University Press, 1986).
} 
sociedad en desarrollo. El rol del Estado en la armonización de coaliciones en competencia responde a la responsabilidad del primero en reorganizar la estructura de actores domésticos para la gestión óptima del bien colectivo. De esta forma la coalición populista prevaleció durante el período de la sustitución de importaciones; la coalición burócrata-élite industrial exportadora-militar prevaleció durante el primer experimento con políticas de industrialización por vía de exportaciones a fines de los setenta. De acuerdo con la lógica de los bienes colectivos, las coaliciones menos favorecidas aún permanecen como beneficiarias del desarrollo económico durante estos períodos dado el carácter no excluyente de los bienes públicos. En otras palabras, como bien público, los beneficios del desarrollo económico poseen un efecto difusión, en tanto que cada individuo tendrá un mejor nivel de vida en un país desarro1lado. No obstante, en la coyuntura actual, el problema de la acción colectiva en la búsqueda de una estrategia desarrollista en el contexto internacional se ha convertido, dadas las características estructurales del sistema internacional, en un problema de instituciones y de responsabilidad que sobrepasa el esquema doméstico de coaliciones políticas. A menos que alguna forma de cooperación internacional institucionalizada surja, no existe ningún mecanismo institucional que imponga la colaboración en los Estados individualmente o que garantice la provisión del bien público. Desde una perspectiva desarrollista y de bienes colectivos, es dentro de este contexto de «cooperación como necesidad» que debe entenderse el resurgimiento de los regímenes de integración regional a través de América Latina.

\section{Consolidación democrática: redefiniendo la arena política}

Una de las más importantes consecuencias de la crisis económica de los 1980 para muchos sistemas políticos en América Latina ha sido la adopción del manejo efectivo de los asuntos económicos como criterio para evaluar la capacidad y el éxito de los gobiernos (Gamarra, 1994). La transición a la democracia en Latinoamérica durante la pasada década por tanto corresponde con la ejecución de programas de ajuste neoliberal luego de las crisis en la balanza de pagos de muchos países de la región. Sería casi imposible intentar comprender la ola de democratización latinoamericana sin examinar la implementación de las políticas neoliberales. Según nota Córdova (1990), la democracia es una condición política necesaria para la liberalización económica en América Latina, ya que los gobiernos autoritarios y las dictaduras militares se adjudicaron tanto el poder económico como el político. De hecho, sería posible incluso pensar en la liberalización como una especie de «democratización económica» limitada, en tanto que la privatización y la desregulación ofreció nuevas oportunidades a los capitalistas latinoamericanos para influenciar la toma de decisiones en materias 
económicas (Conaghan, 1992). Sin embargo, debido a la urgencia del ajuste macroeconómico, las nuevas instituciones democráticas surgidas como resultado de la crisis no tuvieron la oportunidad de pasar por una etapa de aprendizaje político. ${ }^{11}$ Estas instituciones reprodujeron los modelos históricos de protección, adaptados a la ortodoxia neoliberal contemporánea, llegando a lo que Eduardo Gamarra (1994) ha calificado como sistemas políticos «híbridos». Por lo tanto, la democracia latinoamericana parece ser más bien un ejercicio estatista de reacomodar coaliciones domésticas para así poder obtener apoyo para los proyectos neoliberales de ajuste, ya que muy pocas de las características comúnmente vinculadas con el ejercicio de la democracia poliárquica -específicamente la apertura del sistema político y un aumento en el acceso popular al proceso de toma de decisiones- resulta evidente en gran parte del continente. Aún luego que la reforma estatal se convirtiera en el próximo paso luego del ajuste macroeconómico, la arena política ha permanecido notablemente aislada de la sociedad civil. El término "reforma estatal" por tanto se ha visto frecuentemente como sinónimo de privatización, la cual ha sido catalogada por burócratas, elites empresariales y agencias internacionales como fundamental para alcanzar eficiencia económica.

No obstante, la consecución de mayor eficiencia económica se ha transformado en un reto abrumador para las débiles democracias latinoamericanas. Las presiones provenientes de sectores populares que exigen la compensación de necesidades sociales son una prueba para la política democrática, especialmente porque la inestabilidad financiera y los déficits fiscales aún siguen siendo obstáculos de extrema importancia a cualquier aumento en el gasto público. Las recién inauguradas instituciones democráticas en la región aún deben alcanzar suficientes niveles de arraigo social como para establecer su legitimidad en medio de una verdadera explosión de exigencias sociales, típicas de democracias incipientes. Más aún, a pesar de las preferencias populares por regímenes democráticos por sobre los autoritarios, las luchas entre coaliciones domésticas por la distribución de los costos y beneficios de la transición democrática, así como la ineptitud burocrática en el manejo de los asuntos públicos, contribuyen a un ambiente generalizado de inestabilidad política en muchos países latinoamericanos.

Esta situación reduce seriamente el carácter de los posibles incentivos que los Estados latinoamericanos pudieran ofrecer a los muy esperados inversionistas extranjeros, así como de los flujos de capital provenientes de instituciones prestamistas internacionales. La volatilidad de los flujos de capital en las economías latinoamericanas, donde las economías de la región experimentan fluctuaciones masivas en la entrada y salida de capitales (los llamados ciclos de

\footnotetext{
${ }^{11}$ Entre los elementos que comúnmente caracterizan el proceso de aprendizaje político durante el período de democratización figuran la formación de una oposición política leal (en otras palabras, una oposición dispuesta a seguir las reglas del juego político, constitucional y jurídico) así como la seguridad jurídica (en otras palabras, la responsabilidad de los políticos y las políticas ante la ley, así como la transparencia de los procesos de toma de decisiones.)
} 
boom y bust desestabilizan los mercados financieros de la región y restan confianza ante los ojos de inversionistas extranjeros y nacionales, por tanto obstaculizando las posibilidades de estabilidad macroeconómica y crecimiento. ${ }^{12}$ La falta de un ambiente político y empresarial estable podría resultar en un serio daño a la ventaja competitiva de América Latina entre las economías en desarrollo. Sin embargo, en términos del desarrollo mismo, esto significa que a menos que el problema doméstico de acción colectiva para la institucionalización de reformas económicas y de consolidación democrática (en términos de coaliciones y actores políticos nacionales) sea resuelto, los Estados latinoamericanos no están en posición alguna para pasar de la reestructuración económica a una verdadera estrategia de desarrollo. Como se discutirá más adelante, la formación de regímenes de integración regional, particularmente en México y Sudamérica, reduce algunos de los problemas asociados con la acción colectiva, con la consolidación institucional en democracias incipientes, y con la responsabilidad política de los funcionarios públicos por vía de la creación de normas claras y estables de comportamiento político (policy) y económico para los países participantes.

\section{El resurgimiento de la integración latinoamericana: nuevas reglas para un nuevo juego}

La integración latinoamericana se manifiesta durante este período como una nueva oportunidad para avanzar los intereses económicos de la región y, paralelamente, para asegurar un mayor grado de estabilidad en términos de gobernabilidad democrática. Como quedó señalado anteriormente, contrario a acuerdos de cooperación económica dentro de esquemas integracionistas previos, en ningún otro período en la historia de América Latina se ha visto una convergencia de metas, instrumentos, y objetivos para la coordinación de políticas macroeconómicas como en la coyuntura actual. A tono con las recomendaciones neoliberales sobre liberalización y desregulación comercial, un mercado amplificado como resultado de la integración podría proveer incentivos más fuertes para mayor especialización de las economías latinoamericanas en términos de sus ventajas competitivas respectivas y por lo tanto, en términos de intercambios sectoriales (SELA, 1991). La especialización, la competitividad, la eficiencia y el desarrollo tecnológico, son condiciones básicas para el llamado «nuevo industrialismo" en el continente, el cual fomenta una inserción

\footnotetext{
${ }^{12}$ Para un análisis detallado de este fenómeno, así como posibles soluciones e implicaciones para las economías latinoamericanas, véase Ricardo Hausmann y Liliana Rojas Suárez, Volatile Capital Flows: Taming Their Impact on Latin America (Washington, D.C.: Banco Interamericano de Desarrollo, 1996).
} 
selectiva y programada en los mercados mundiales para las economías latinoamericanas en proceso de reestructuración, mediante una estrategia muy similar a aquélla seguida por los países del Asia oriental (SELA, 1991).

El proceso por el cual un país se mueve de niveles básicos a niveles más avanzados de producción industrial como búsqueda de la mencionada inserción ventajosa en los mercados mundiales corresponde con lo que Gary Gereffi llama el modelo de desarrollo por vía de «cadenas globales de [comodificación]" (Gereffi, 1994). Este modelo se basa en la premisa de que la globalización de los flujos de capital y la trasnacionalización de la producción industrial han reducido la utilidad del Estado como punto de referencia para el análisis de la efectividad de las estrategias de desarrollo. Debido a que las posibilidades de desarrollo económico de un país están afectadas, en gran medida, por la manera en que dicho país se inserta en industrias globales (por ejemplo, en cadenas de producción multinacional) (Gereffi, 1994), las opciones para el desarrollo no sólo deben ser evaluadas en términos de preferencias estatales, sino más bien en términos de la inserción de dicho país en cadenas globales de "comodificación" (en otras palabras, en redes de mano de obra y producción vinculadas a bienes específicos) por vía de la especialización productiva, así como de una gerencia y mercadeo efectivos.

De acuerdo con esta condición, los esquemas de integración podrían convertirse en un instrumento gubernamental para estimular y coordinar la especialización industrial y el desarrollo tecnológico de América Latina a nivel regional. Al asegurar condiciones infraestructurales, burocráticas y legales que sean transparentes y eficientes para la implementación del «nuevo industrialismo» y de la apertura económica, la integración bien podría reducir los costos de transacción que conlleva la transformación de la plataforma industrial latinoamericana. Debido a que el «nuevo industrialismo» no puede ejecutarse sin antes lograr serias transformaciones tecnológicas, un mercado amplificado podría crear mejores condiciones para aunar recursos y para lograr mejor colaboración internacional en materia de investigación científica, así como para negociar transferencias tecnológicas de centros industriales avanzados así como dentro de la región misma. ${ }^{13}$ Un acuerdo de integración también podría reducir los costos de protección de tecnologías incipientes que hayan sido favorecidas por los gobiernos nacionales, de forma similar a la forma en la que los Estados practican el comercio estratégico con miras a la protección de industrias emergentes.

\footnotetext{
${ }^{13}$ Claro, esto está sujeto a cuestiones de cooperaciōn entre los paîses que componen el esquema de integración. Para resolver dichas cuestiones es necesario establecer mecanismos de negociaciôn regional transparentes, asi como mecanismos de solución de controversias que se adecuen a las preferencias politicas y económicas de sus miembros. Esto, que ha sido motjvo de disputas entre paîses en la Uniốn Europea, se ha visto también en el caso del MERCOSUR, específicamente en términos de los intereses brasileños frente a los de sus socios.
} 
En términos de sus efectos en el sistema democrático, aunque la formación de un esquema de integración puede no llevar inmediatamente a la expansión del sistema político, sobre todo a un sistema político más abierto, es posible asumir que el grado de voluntad política requerido para la implementación exitosa de la integración regional puede servir como agente «neutralizador» en términos de los conflictos y las competencias políticas domésticas. Esta perspectiva se fortalece precisamente a partir del hecho que el acceso a un acuerdo de integración automáticamente implica la delegación de algún grado de soberanía nacional (Smith, 1993). La arena política de un país inevitablemente sufrirá algún grado de reconfiguración, tanto en términos de actores como de mecanismos e instituciones, a partir de esta cesión de soberanía. Según lo evidenciado en el caso de NAFTA, el acuerdo mismo de integración puede sin embargo convertirse en el centro de un agitado debate político nacional. ${ }^{14}$ Incluso los beneficios de la integración para los sectores populares frecuentemente quedan en entredicho. En muchos casos incluso pueden no ser evidentes de primera instancia, particularmente porque, como en muchas democracias incipientes, el proceso de negociación entre actores regionales continúa siendo un proceso elitista. ${ }^{15}$ Más aún, según observa Córdoba (1990), la institucionalización de la cooperación regional no necesariamente conduce a mayor eficiencia gubernamental, ya que lo que en realidad podría suceder es la sustitución de una burocracia doméstica por una internacional. ${ }^{16}$

No obstante, es posible señalar dos puntos con relación al vínculo entre democracia y los procesos de integración regional. Un punto es que, como instrumento desarrollista, la integración exitosa exige una cantidad considerable de aprobación política a priori, la cual ninguna de las estrategias desarrollistas aplicadas a través de América Latina durante los últimos veinte años ha sido capaz de alcanzar. Esto se debe a que ha habido un cambio en las reglas del juego donde la legitimidad política de las decisiones económicas ha pasado a ser materia de debate público en muchos países latinoamericanos. Segundo, si la integración se asume no sólo en términos de apertura comercial y desregulación (en otras palabras, un acuerdo de libre comercio) sino como un instrumento necesario para el «nuevo industrialismo», esto podría significar

\footnotetext{
${ }^{14}$ Este punto resulta de sumo interés, sobre todo desde una perspectiva comparada, si se examinan los diversos acuerdos de integración en América Latina. Como punto de referencia, y en contraposición a la experiencia del NAFTA, la ratificación del MERCOSUR ha sido uno de los procesos recientes donde más consenso político ha existido en Argentina, Uruguay y Brasil. De hecho, en el caso de Uruguay, el Tratado de Asunción (origen del MERCOSUR) fue ratificado por unanimidad en la cámara baja, y por casi unanimidad en la cámara alta. Ningún partido político uruguayo o argentino ha usado el MERCOSUR como purto de debate en contiendas electorales.

${ }^{15}$ Para un estudio sobre la experiencia mexicana en la negociación del NAFTA, vêase Manuel Pastor (1994).

${ }^{16}$ Esta posibilidad ha quedado muy clara entre los responsables de la toma de decisiones en la integración latinoamericana actual, sobre todo ante la sombra del fracaso de los experimentos integracionistas de los '6o.
} 
una estrategia de desarrollo más inclusivo, en tanto que proyectos expansionistas de transformaciones socioeconómicas podría ser puesta en marcha mediante la reconfiguración industrial. ${ }^{17}$

Sin embargo, el proceso y la condición de la integración latinoamericana no es asumido igualmente por analistas o por políticos. No se trata meramente de un problema tipológico, ya sea en términos sistémicos (por ejemplo, qué significa «inserción internacional adecuada», o cómo un esquema de integración podría «encajar» en la estructura de relaciones de poder en el sistema internacional) o en términos económicos (por ejemplo, la clasificación de acuerdos de integración según el grado de liberalización comercial o de coordinación económica). Se trata más bien de un dilema procesal, específicamente en cuanto a la cuestión del convenio y la convergencia política. De hecho, el SELA (Sistema Económico Latinoamericano) caracteriza la integración como un umbral en la armonización de políticas económicas, luego de que se haya embarcado en un proceso previo de convergencia política. CEPAL, sin embargo, es más moderada en sus sugerencias con relación a coordinación de políticas. CEPAL (1992) resalta que los países latinoamericanos carecen de experiencia en materias de colaboración económica, y mucho menos en asuntos de coordinación. Más aún, CEPAL apunta que debido a los bajos niveles de interdependencia en América Latina (según lo demuestra, por ejemplo, por la baja proporción del comercio interregional como porcentaje del total de actividades comerciales latinoamericanas aún dentro de los acuerdos de libre comercio existentes) no debería haber expectativas muy altas sobre los beneficios inmediatos, o aún sobre la viabilidad, de coordinación macroeconómica. Su recomendación para una agenda integracionista se basa en la flexibilidad y la contingencia por encima del compromiso institucional y la formalidad. En esta aparentemente temprana etapa (1991), la CEPAL no cree que cualquier experimento de coordinación económica más allá del asunto comercial sea exitosa. Esta postura es, interesantemente, diferente a la propuesta del SELAA, la cual está enfocada hacia la construcción de una infraestructura productiva adecuada por vía de la cooperación y la coordinación regional, para así poder crear un ambiente comercial latinoamericano cualitativamente diferente a épocas anteriores.

Con relación a debates más tradicionales sobre cooperación en relaciones internacionales, se puede ver esta disyuntiva organizativa en términos de intereses individuales y comporta-

\footnotetext{
${ }^{17}$ Un caso interesante de este fenómeno es la transformación del sector productivo en el Uruguay desçe que comenzó el MERCOSUR. La economía uruguaya está compuesta en un $99.4 \%$ por empresas pequeñas y medianas, las cuales, ante los retos de ta unión aduanera con Brasil y Argentina, han formado consorcios de exportación que les permitan competir más favorablemente con sus vecinos. Asimismo, las industrias uruguayas están pasando por un proceso de reconversión productiva donde los sectores menos competitivos están siendo eliminados, y los recursos liberados se están empleando en sectores donde Uruguay posee una ventaja comparativa. Para un examen detallado de este fenómeno, véase Antonio Maeso Ruiz y Dalton Daemon, Consorcios de exportación en Uruguay: experiencias internacionales (Montevideo: Fundaciôn de Cultura Universitaria / Cámara de lndustrias del Uruguay, 1997).
} 
miento estatal. La CEPAL reconoce explícitamente que los intereses de los Estados prefiguran cualquier acuerdo comercial, posiblemente dada la insuficiencia en la confianza mutua entre los países latinoamericanos. ${ }^{18}$ En este sentido, resulta obvio por qué la CEPAL, a un nivel inicial, hace recomendaciones sustanciales sobre coordinación comercial y no macroeconómica. La CEPAL implícitamente reconoce que la institucionalización no es prerequisito para el surgimiento de coordinación internacional y de "integración». Por tanto, una vez que los países latinoamericanos aprendan a partir de sus experiencias en colaboración comercial, la coordinación formal de políticas económicas sería mucho más factible (tal y como lo señalarían las teorías ortodoxas de integración regional). Se puede notar cómo el tema de irreversibilidad queda apartado como otro debate, que depende del «rol subsidiario» de la coordinación macroeconómica para el avance de la integración en general (CEPAL, 1992). ¿Pero cómo se puede llegar a la cooperación sin un aparato institucional legítimo que enmarque el proceso de coordinación? Aquí resurge la cuestión de la acción colectiva y la integración, en términos de instituciones y bienes colectivos: si se espera que la integración produzca una serie de bienes colectivos -como por ejemplo, la reducción en los costos de transacción del comercio y la cooperación internacional como resultado de normas comerciales claras, mejoras infraestructurales y "transparencia" en las políticas económicas- ¿cómo se puede asegurar la acción colectiva y prevenir el problema del free rider (Olson, 1982) sin cohesión institucional? Ya que muy posiblemente los intereses estatales anteceden la cooperación, según el paradigma realista de las relaciones internacionales, por tanto la tendencia natural del comportamiento estatal es maximizar el beneficio propio y extraer beneficios del vecino. La CEPAL esta muy consciente de esta posibilidad; de hecho, sugiere una serie de "medidas administrativas" para así reducir la incertidumbre acerca del comportamiento de los Estados, y por lo tanto acrecentar la "claridad de normas" que forma parte de la reducción en los costos de transacción aludida anteriormente (CEPAL, 1994). ¿Pero cómo se puede concebir e imponer estas "medidas" sin sanciones institucionales? Para una respuesta posible a este dilema de coordinación y acción colectiva, acudimos ahora a la perspectiva analítica proveniente de la teoría de regímenes internacionales.

\footnotetext{
${ }^{13}$ Para un estudio sobre medidas de confianza mutua en América del Sur y América Central, véase Augusto Varas (ed.) Medidas de confianza mutua en América Latina (Santiago, Chile: FLACSO, 1994). Este reconocimiento de la CEPAL sobre los intereses estatales coincide interesantemente con la perspectiva intergubernamentalista sobre los procesos de integración, a la cual se aludió anteriormente en este trabajo.
} 


\section{La integración, ganancias relativas versus ganancias absolutas, y la cooperación: perspectivas de la teoría de regímenes}

Contrario a instancias aisladas de colaboración interestatal, un régimen es un "contrato voluntario" que permite el establecimiento de expectativas mutuas, así como el desarrollo de una relación de trabajo entre varios actores internacionales (Keohane, 1984). El énfasis en expectativas mutuas y en relaciones de trabajo es central al surgimiento de regímenes internacionales. En términos de la cooperación institucionalizada, las posibilidades de interacción reiterada y la condición de responsabilidad (mediante normas y sanciones) hacen de la formación de regímenes una manera deseable de organizar las relaciones interestatales, sobre todo cuando una meta común es perseguida simultáneamente por varios Estados. De hecho, en su análisis de los regímenes internacionales, Arthur Stein (en Baldwin, 1993) resume así las condiciones que llevan a las naciones a formar tales instituciones: «Las naciones soberanas poseen un incentivo racional para tomar decisiones conjuntas cuando confrontan problemas de intereses comunes o de aversiones comunes. En estos contextos, actores [internacionales] interesados en el beneficio propio renuncian a la toma de decisiones individual y construyen regímenes». ${ }^{19}$ En otras palabras, cuando Estados característicamente egoístas confrontan situaciones o crisis para las cuales una respuesta individual sería adecuada, pero no óptima (dilema de interés común), o cuando los Estados poseen estrategias reactivas o incidentales, así como un interés común en la prevención de un resultado indeseable (dilema de aversión común), estos Estados se reúnen y forman un régimen.

De estos dos tipos, el dilema de intereses comunes es particularmente interesante para una discusión sobre integración económica. El dilema de intereses comunes es característico de aquellas situaciones donde el bien colectivo internacional no puede ser alcanzado en términos de optimalidad de Pareto ${ }^{20}$ a menos que los Estados desechen sus estrategias individuales (las cuales conducen a una condición de equilibrio, no de optimización y que controlen sus tendencias de aprovecharse o «robar» ("free-ride") del bien colectivo (en otras palabras, el típico Dilema del Prisionero). Nótese que esto aún sigue siendo un argumento de intereses nacionales: los Estados se reúnen para formar un régimen con su interés propio en mente. Dejando a un lado la cuestión de la distribución de poder entre los actores que componen el régimen, el ángulo analítico para comprender la cooperación institucionalizada no es tan sólo cuestión de intereses, sino también la de preferencias: ante una situación de absolutas, ¿bajo qué arreglo institucional internacional pueden los Estados optimizar sus estrategias e intereses individuales?

\footnotetext{
29 Traducción del autor.

${ }^{20}$ El punto de optimalidad Pareto se refiere a la asignación de recursos o bienes de forma tal que nadie puede aumentar sus beneficios sin a la vez disminuir los beneficios ajenos.
} 
Uno de los debates contemporáneos en el estudio de las relaciones internacionales se centra precisamente en la cuestión de las ganancias absolutas versus las ganancias relativas como el asunto que articula la interacción entre los Estados ${ }^{21} \mathrm{Si}$ el escenario de las relaciones internacionales se concibe como un juego de suma cero, en el cual las ganancias del otro lado se entienden como pérdidas para el lado propio, es casi imposible concebir que existan intereses comunes, mucho menos la posibilidad de cooperación. Sin embargo, esto no siempre es así, particularmente cuando preocupaciones más apremiantes o inmediatas sobrepasan la preocupación por poder y por capacidades relativas. En este sentido, es posible entender el resurgimiento de la concertación latinoamericana, y el lanzamiento de la regionalización económica como un proyecto común para los 1990 , como resultado de abrumadores retos comunes como la deuda externa, la reestructuración macroeconómica, el conflicto regional, la inestabilidad política y el desarrollo.

Por tanto, la formación de regímenes de integración como parte de un proyecto desarrollista (ya sea «nuevo industrialismo» o «regionalismo abierto») puede entenderse como una respuesta a las exigencias del desarrollo en un contexto internacionalizado, así como a la ausencia, o en el mejor de los casos inexperiencia, de colaboración y coordinación entre los Estados latinoamericanos. Es por esto que la institucionalización es tan importante para medir la efectividad de los esfuerzos de regionalización. Al imponer sanciones a los desertores, así como otras reglamentaciones al comportamiento individual, los regímenes contribuyen a asegurar la condición de «irreversibilidad» que los analistas de CEPAL han afirmado continuamente como necesarias para la formulación de estrategias de desarrollo a largo plazo. El potencial para modificaciones en el comportamiento es característico de los regímenes internacionales y no de otras formas de cooperación internacional; las Naciones Unidas y el Grupo de Río siendo los ejemplos más sobresalientes de esta última. La reglamentación al comportamiento individual característica de los regímenes de integración sirve como instrumento para asegurar la cooperación y la conformidad, así como para evitar la explotación entre Estados, al limitar el ámbito de opciones estatales ${ }^{22}$ y de estrategias de política exterior. ${ }^{23}$ También sirve como catalítico supranacional y como posible agente neutralizador de algunos actores domésticos -especialmente relevante para las nuevas democracias latinoamericanas- cuyos intereses dispares (y frecuentemente conflictivos) podrían potencialmente resultar en inestabilidad política interna. Un ejemplo clásico de esta característica de los regímenes de integración es la

\footnotetext{
21 Entre los volúmenes mâs sobresalientes en este debate figuran Robert o. Keohane (ed.), Neorealism and its Critics (New York: Columbia University Press, 1986) y David Baldwin (ed.), Neorealism and Neoliberalism (New York: Columbia University Press, 1993). ${ }^{22}$ Por ejemplo, la tentación de algunos líderes de impulsar políticas distributivas de corte populista, las cuales proveen ganancias electorales inmediatas pero que afectan la credibilidad de las reformas económicas. Véase Barbara Geddes, Politician's Dilemma: Building State Capacity in Latin America (Berkeley, CA: University of California Press, 1994).

${ }^{23}$ Por ejemplo, cambios en los patrones e incentivos a la inversión extranjera y a la competencia industrial.
} 
formación del Tratado de Roma en 1957 y sus efectos estabilizadores en la arena política de las democracias emergentes en Alemania e Italia (Córdova, 1990). El caso de la negociación para la entrada española a la Comunidad Europea luego de la reinstauración de la democracia en ese país es un ejemplo más reciente de este fenómeno. Más aún, debido a que los regímenes frecuentemente conllevan un conjunto «estandarizado» de reglas y normas, tanto explícitas como implícitas, también refuerzan el patrón de preferencias y expectativas iniciales que dieron lugar al surgimiento del régimen en primera instancia. Según los proponentes de la teoría de regímenes, este proceso de formalización de reglas internacionales contribuye a una mayor convergencia interestatal, de manera que las divergencias en materia de preferencias e intereses se van manejando a través de los parámetros de la organización internacional, a la cual los estados han sido paulatinamente socializados. En otras palabras, los estados aún pueden beneficiarse de la provisión de un bien colectivo, aún cuando sus intereses hayan sufrido transformaciones. En el caso latinoamericano, el proyecto de desarrollo económico puede entenderse en la manera en que ha creado preferencias distintas entre los actores regionales por tanto las diferencias en los varios grupos de integración a través del continente), pero no necesariamente un conjunto de intereses diferentes, hasta el punto que la colaboración entre estos grupos sea imposible. La evidencia proveniente de conversaciones entre el CARICOM (Comunidad Caribeña) y el MCCA (Mercado Común Centroamericano), la participación conjunta de México en el NAFTA y en el Grupo de los Tres, así como el interés boliviano por entrar como socio pleno del MERCOSUR, tiende a apoyar esta contención. 


\section{Comentarios finales}

El propósito principal de este trabajo ha sido vincular el surgimiento de esfuerzos integracionistas en América Latina luego de la Guerra Fría, por vía de regímenes institucionalizados, a un conjunto particular de límites, retos y oportunidades sistémicas dentro de los cuales la cooperación regional es considerada como la mejor forma de alcanzar algún grado de éxito en los intentos por alcanzar desarrollo económico. Varios puntos de vista de la teoría de regímenes se han usado para ilustrar por qué las naciones latinoamericanas se han embarcado en proyectos de regionalización económica sin las precondiciones económicas, y frecuentemente políticas, apropiadas que los economistas normalmente sugieren como necesarias para una integración efectiva.

Sin embargo, este ejercicio no ha sido exhaustivo en 10 absoluto. Hay numerosas variables interventoras en las esferas políticas domésticas e internacionales, tales como la asimetría entre los actores regionales, lo que Baldwin (1993) llama «técnicas de habilidad política», la estructura de las coaliciones domésticas, y la influencia de los líderes políticos, que han sido explícitamente evadidas con el propósito de proveer una visión parsimónica de la integración, de manera que pueda haber un debate sobre estos temas. Este trabajo tampoco ha tratado de identificar una solución definitiva al problema de la coordinación internacional, especialmente en el ámbito macroeconómico, ya que los asuntos de cooperación interestatal son extremadamente más complicados que lo que modelos simples pueden explicar. Sin embargo, al proponer una lógica de intereses y preferencias comunes, me parece haber contribuido a un análisis más riguroso y sistemático del carácter y del proceso de la integración latinoamericana. Desafortunadamente, debido a que hay tanto en juego, y ya que «hemos permitido que nuestras herramientas conceptuales se enmohezcan», en palabras de Bárbara Stallings, muchas discusiones académicas y políticas de este intrincado proceso parecen no haber capturado la complejidad de la regionalización e integración económica. Precisamente debido a que hay tanto por medio, espero que este trabajo sea una herramienta útil para explorar esta complejidad en nuestra agenda regional. 


\section{Bibliografía}

Baldwin, David A. 1993. Neorealism and Neoliberalism: The Contemporaly Debate. New York: Columbia University Press.

Caputo, Dante M. and Jorge F. Sábato. 1991. «Perspectivas de la integración político-económica continental. La integración de las democracias pobres: oportunidades y peligros.» [Perspectives on Continental PoliticEconomic Integration. Integration Among Poor Democracies: Opportunities and Dangers]. Estudios Internacionales (Santiago) $94:$ 194-208.

Conaghan, Catherine. 1992. «Capitalists, Technocrats and Politicians: Economic Policy Making and Democratization in the Andes. «En Issues in Democratic Consolidation: The New South American Democracies in Comparative Perspective, ed. Scott Mainwating, Guillermo O'Donnell y J. Samuel Valenzuela. Notre Dame, IN: Notre Dame University Press.

Córdova, Arnaldo. 1990. «Modernización y desarrollo» En Desarrollo y Democracia, ed. Margarita López Maya. Caracas: Nueva Sociedad.

CEPAL. 1992. Ensayos sobre Coordinación de Políticas Macroeconómicas. Santiago: CEPAL.
CEPAL. 1994a. Políticas para mejorar la inserción en la economía mundial. Santiago: CEPAL

CEPAL. 1994b. El regionalismo abierto en América Latina y el Caribe. Santiago: CEPAL.

Frohmann, Alicia. 1990. Puentes sobre la turbulencia: La concertación política latinoamericana en los 80. Santiago: FLACSO.

Gamarra, Eduardo. 1994. «Market-Oriented Reforms and Democratization in Latin America: Challenges of the 1990's.» En Latin American Political Economy in the Age of Neoliberal Reform, ed. William C. Smith, Carlos H. Acuña and Eduardo A. Gamarra. New Brunswick, N.J.: Transaction Publishers.

Gereffi, Gary. 1994. «Contending Perspectives on Regional Integration: Development Strategies and Commodity Chains in Latin America and EastAsias. Presentado en el XVIII Congress de la Latin American Studies Association (LASA), Atlanta, Georgia.

Haggard, Stephan. 1990. Pathways from the Periphery: The Politics of Growth in the Newly Industrializing Countries. Ithaca, N.Y.: Cornell University Press.

Keohane, Robert. 1984. After Hegemony: Cooperation and Discord in the World Political Economy: Princeton, NJ: Princeton University Press. 
Lechner, Norbert (ed.) 1991. Capitalismo, democracia y reformas. Santiago: FLACSO.

Manzetti, Luigi. 1993. «The Political Economy of MERCOSUR.» Journal of Interamerican Studies and World Affairs 35 (Invierno '93-'94): 101-141.

Moravcsik, Andrew. 1993. «Armaments Among Allies: European Weapons Collaboration, 1975-1985.» En DoubleEdged Diplamacy: International Bargaining and Domestic Politics, ed. Peter B. Evans, Harold K. Jacobson and Robert D. Putnam. Berkeley, CA: University of California Press.

North, Douglass C. 1981. Structure and Change in Economic History. New York: W.W. Norton \& Co.

Olson, Mancur. 1982. The Rise and Decline of Nations: Economic Growth, Stagflation, and Social Rigidities. New Haven,, CT: Yale University Press.

Oye, Kenneth A. (ed.) 1986. Cooperation Under Anarchy. Princeton, N.J.: Princeton University Press.

Pastor, Manuel y Carol Wise. 1994. «The Origins and Sustainability of Mexico's Free Trade Policy.» International Organization 48 (3): 459-489.
Pierson, Paul. 1995. 'The Path to European Integration: A Historical Institutionalist Perspective». Harvard University. Mimeo.

Putnam, Robert. 1988. «Diplomacy and Domestic Politics: The Logic of Two-Level Games.» International Organization 42: 427-460.

Roett, Riordan. 1991. «El comercio no es suficiente». En Cambios en el ambiente comercial hemisférico: Oportunidades y obstáculos, ed. Mark B. Rosenberg. Santiago: FLACSO.

Rojas Aravena, Francisco (ed.) 1993. América Latina y la Iniciativa para las Américas. Santiago: FLACSO.

Smith, Peter H. (ed.) 1993. The Challenge of Integration: Europe and the Americas. New Brunswick, N.J.: Transaction Publishers.

Stallings, Barbara. 1994. «El nuevo contexto internacional del desarrollo: América Latina desde una perspectiva comparada.» En El Cono Sury las transformaciones globales, ed. Francisco Rojas Aravena and William C. Smith. Santiago: FLACSO. 